\title{
Assistive technologies for the new generation of senior citizens: the SHARE-it approach
}

\section{U. Cortés* , C. Barrué and A.B. Martínez}

Technical University of Catalonia, Jordi Girona 1-3, Barcelona, 08031, Spain

E-mail: ia@1si.upc.edu

E-mail: cbarrue@1si.upc.edu

E-mail: antonio.b.martinez@1upc.edu

*Corresponding author

\section{Urdiales}

University of Malaga,

Campus de Teatinos, Malaga, 29071, Spain

E-mail: cristina@dte.uma.es

\section{F. Campana}

Azienda Unita Sanitaria Locale Roma B Centro Assistenza Domiciliare (CAD RMB),

Via della tenuta di Torrenova, 138,

Rome, 00133, Italy

E-mail: fcampana@tiscali.it

\section{R. Annicchiarico and C. Caltagirone}

Fondazione Santa Lucia,

Instituto di Ricovero e Cura a Carattere Scientifico,

Via Ardeatina 306, Roma, 00179, Italy

E-mail: r.annicchiarico@hsantalucia.it

E-mail: c.caltagirone@hsantalucia.it

\begin{abstract}
In this paper, we present the SHARE-it approach to the integration of agent technology with other existing technologies to build specific intelligent and semi-autonomous assistive devices for older persons and people with disabilities (both cognitive and/or motor). In particular, we explored the benefits of the concept of situated intelligence to build mobility platforms and other artefacts that enhance the autonomy of the target user group in their daily life so that they can be self-dependent enough to autonomously live in their preferred environment.
\end{abstract}

Keywords: assistive technologies; intelligent agents. 
Reference to this paper should be made as follows: Cortés, U., Barrué, C., Martínez, A.B., Urdiales, C., Campana, F., Annicchiarico, R. and Caltagirone, C. (2010) 'Assistive technologies for the new generation of senior citizens: the SHARE-it approach', Int. J. Computers in Healthcare, Vol. 1, No. 1, pp.35-65.

Biographical notes: Ulises Cortés is the Artificial Intelligence Professor at Software Department at the Technical University of Catalonia, Barcelona, Catalonia. He obtained his $\mathrm{PhD}$ from Technical University of Catalonia, Barcelona in 1984. Since 1984, he has been researching in knowledge representation and agents technology.

Cristian Barrué obtained his MSc from the Technical University of Catalonia, Barcelona in 2006. His main research interests are in agent technologies and machine learning applied to assistive technologies and e-health.

Antonio B. Martínez received his degree in Electrical Engineering in 1984 from the School of Engineering of Barcelona (ETSEIB-UPC) followed by his $\mathrm{PhD}$ in Computer Science in 1988 (FIB-UPC). He is now an Assistant Professor in the Automatic Control and Computer Engineering Department at the Universitat Politècnica de Catalunya. His research area is in the field of robotics and computer vision, mainly oriented to robotics applications. $\mathrm{He}$ is currently leading projects on mobile robots applied to intelligent platform either at factories or applied as assistive robotic aid for elderly people, such as walker, wheelchair or stretcher.

Cristina Urdiales received her MSc in Telecommunication Engineering from the Universidad Politécnica de Madrid (UPM), Spain and her PhD from the Universidad de Málaga (UMA), Spain, where she is currently a Lecturer. She works in the ISIS group and her research is focused on robotics and computer vision. Her current interests are in assistive robotics and shared control.

Fabio Campana is a Geriatrician committed in clinical activity, working on clinical application of EHRs to geriatric patients and ICT solutions to support the elderly in ADLs. He was the Medical Manager of the EU project K4CARE and the Chief of a research unit of the EU project SHARE-it. He took part in the epidemiological branch of Progetto Finalizzato Invecchiamento and multicentric epidemiological studies. He was a Professor of Geriatrics at the School of Physiotherapy Un. La Sapienza (Rome).

Roberta Annicchiarico is a Geriatrician. She is a Medical Assistant at IRCCS Fondazione S. Lucia, Rome since 2002. She is a contracted Professor of Internal Medicine with focus on Geriatrics at the School of Physiotherapy, University 'Tor Vergata', Rome since 2004. She is a contracted Professor of Geriatrics at the school of Nurse, University 'Tor Vergata', Rome since 2005. She is participating in several European project. Her main research interests are in disability and possible clinical applications of new technologies.

Carlo Caltagirone is a Full Professor of Neurology at the Medicine Faculty at Università 'Tor Vergata' in Rome and the Chief of the Neurology and Neurological Rehabilitation Division at the 'Fondazione Santa Lucia'. Since 2002, he is the Scientific Director of the 'Fondazione Santa Lucia'. $\mathrm{He}$ participated in the committee which defined the Italian guidelines on diagnosis and management of Alzheimer disease and dementias. $\mathrm{He}$ participates in the Board of the Italian Network on Alzheimer Disease 
(ITINAD), leading the research group on mild cognitive impairment. His main research interests are in clinical neurology, clinical and behavioural neuropsychology and neurorehabilitation.

\section{Introduction}

The main goal of the SHARE-it project (SHARE-it, 2007) was to contribute to the development of the next generation of intelligent and semi-autonomous assistive devices for older persons and people with disabilities (both cognitive and/or motor). The basic assumption in this research effort is that the target population by using these supporting devices could be self-dependent enough as to autonomously live in their community, staying at home as long as possible with a maximum safety and comfort; this possibility would increase their quality of life and, at the same time, delay their institutionalisation. At least in part, how well these elders live and their abilities to maintain independent life styles will depend on their health and the degree to which they have remained totally able or frail or disabled. This in turn, will depend to at least some extent on how well the artificial and built environments, in which they live, conform to their needs and their age-related losses in abilities and somatic integrity.

Disability and frailty are common geriatric conditions; these common conditions hold substantial implication for functioning of older people and for their quality of life. Disability and frailty have common characteristics: both are rather prevalent in older population, of multifactorial nature and share some risk factors and pathophysiological mechanisms (Inouye et al., 2007). Despite multiple and often overlapping definitions of disability and frailty, both are common clinical characteristics of aged individuals though not identical. The geriatric syndrome of frailty is described as status of global impairment of physiological reserves involving multiple organ systems (Topinkova, 2008). The clinical correlate of frailty manifests as increased vulnerability, impaired capability to withstand intrinsic and environmental stressors and limited capacity to maintain physiological and psychosocial homeostasis. Frailty includes such alterations as decreased mobility, strength and responsiveness to external and internal stressors, low levels of physical activity and weight loss, along with psychosocial perceptions of health and well-being.

Currently, disability is defined as difficulty or dependency in carrying out activities necessary for independent living, including roles, tasks needed for self-care and household chores and other activities important for a person's quality of life (Fried et al., 2004). The natural course of frailty is progressive, increasing the risk of comorbidity and disability over time. In fact, findings support the hypothesis that frailty cause disability, independent of clinical and subclinical diseases. The syndrome of frailty may be a physiologic precursor and etiologic factor in disability, due to its central features of weakness, decreased endurance and slowed performance (Fried et al., 2001).

There are consequently two important targets, that we subscribe, in future research focused on ageing population: 
- to develop consistent and valid methods for assessing frailty and disability in order to individuate the main areas in which this target population needs help

- to design environments appropriate for the proportion of elders who are increasingly frail and disabled but whose life expectancy continues to grow.

Different groups of elders are characterised by large variations in health, well-being, disability and healthcare needs. Assistive technologies' (AT) major purpose is represented by the possibility of assisting people - elderly and/or disabled - to stay or to get back to their home. Thus, a target population - in terms of functional, social and clinical features - has to be individuated so that a number of individuals as large as possible can achieve benefits as effectively as possible. According to these premises, our target population is operatively individuated through the assessment of the presence of:

- Mild functional impairment: Since older and/or disabled populations are made up by individuals who present widely different and heterogeneous functional profiles, we need to identify a set of this population with well-defined features. Considering the more prevalent diagnosis in this group of persons (stroke, Parkinson's disease, Alzheimer's disease), impairments range from extremely mild (people able to walk with a cane or affected by such a mild memory loss that allows them to live on their own) to extremely severe (persons bedridden or completely unable to understand a simple order). In the first case, we are in the realm of prevention and they do not need assistance. In the second case, we are talking about people who need palliative care or institutionalisation; the level of assistance need is too ambitious for a single project as SHARE-it. People suffering of a mild functional impairment, those in the middle area of impairment, are expected to have the best results through the use of proper AT and are the target of the assistive intervention.

- Possibility of changing functional profile: Mild disability is characterised by the possibility of increasing the functional level according to proper assistive or rehabilitative interventions. Users of a flexible assistive device can benefit from its adaptation so that it could help to improve their condition as a result of rehabilitation treatment, or can prevent the decrease of their autonomy due to intervening conditions or diseases.

This paper considers the definition of SHARE-it users and the activities of daily living (ADL) where SHARE-it services and hardware devices can offer some kind of personalised support to them. The considered elderly population is assessed through the multidimensional geriatric approach. Users are classified on the basis of their functional impairment as suggested by the World Health Organization (WHO). Furthermore, since one of the parameters that affect the user's performance in a specific task is the emotional reaction, we are considering the alarm reaction as an aspect to be detected and to be used as the basis on the elaboration of the decision to give or not support to the user. Also, it will be used to decide which kind and amount of support to give to each individual.

\subsection{Plan of paper}

In Section, 2 a state-of-the-art of AT in SHARE-it context is presented. Both assistive wheelchairs and walkers are deeply considered as SHARE-it objectives centred on 
mobility issues. In Section 3, we discuss the general problem where SHARE-it (SHARE-it, 2007) is willing to contribute with its solutions. That is, we try to make clear the potential of technological interventions - hardware and software - to support or restore impaired physical and/or cognitive functions.

For a large part of the elderly, abilities to perform ADL tasks are essential and critical for living autonomously. In Section 4, we extensively explain our agent-based approach to give support to elders with special functionalities to live autonomously in their preferred environment. In special, we detail the architecture of the multi-agent system (MAS). In this section, we make an emphasis on one of the SHARE-it mobility platforms the $i$-Walker (see Section 4.3).

In Section 5, we introduce one of the collaborative techniques that we implemented as a way of allow the personalisation of the mobility platforms; in this case, we show the application to a power wheelchair. This section shows the basis for the experiments that we are carrying with volunteer inpatients at the Fondazione Santa Lucia (FSL), in Rome.

In Section 6, we discuss some the experiments we performed and the basic settings that we used during the experimentation. In Section 7, we present our conclusions and discuss our point of view.

\section{State-of-the-art}

The SHARE-it approach has to do with several research areas, all of them very active in the scientific community and strongly supported by private and public initiatives. The three main research areas involved are:

1 AT for elders

2 environment integration of those AT with the environment the elder is living

3 e-Inclusion as a final objective of the solutions developed.

\subsection{Assistive technologies}

The EU scientific community have shown an increasing interest in the e-health field. Targeted multidisciplinary European programmes have already been established to encourage integration between different basic disciplines and serve as strong drivers for the whole field of research and service development around ageing. These projects operate in a challenging environment, producing innovative ideas to support the needs of the sensitive group of elderly people (Kamel-Boulos et al., 2009). Researchers focused on how to deliver quality care to a rapidly growing population of older adults. AT for elders address elders' physical problems, cognitive problems or both.

AT for physical problems have been usually associated with supported mobility. The first autonomous wheelchairs like the proposed by Connell and Viola (1990) simply provided a basic set of primitives like AvoidObstacle, FollowWall and PassDoorway to assist the person in difficult manoeuvres. In most cases, these primitives were manually selected by the users. Further systems like NavChair (Simpson and Levine, 1998), Tin Man (Miller and Slack, 1995) or Smartchair (Rao et al., 2002) were mostly based on the subsumption architecture (Brooks, 1986). In these cases, rather than manual selection, events detected by onboard sensors triggered one or several behaviours. These 
behaviours were merged, according to some rules, into an emergent one. The main difference among these systems is how behaviours are implemented. In some cases, the user does not guide the chair at all, but simply provides a destination (Simpson and Levine, 2002). Some of these systems let the user override control whenever he/she wants to (Miller and Slack, 1995). For safety reasons, in some cases, the wheelchair may also override the human use and select a behaviour on its own (Simpson and Levine, 1998; Gomi and Griffith, 1998).

More recently, research has given much attention to assistive wheelchairs like sensor-aided intelligent wheelchair navigation system (SENARIO) (Katevas et al., 1997), VAHM (Bourhis et al., 2001), Wheelesley (Yanco, 1998), Rolland, Navchair or Smartchair. All these systems share some common features: a set of sensors, some processing unit and the software to decide what to do. Homes are not naturally adapted to wheelchairs and may present natural barriers like narrow doors, corridors or structure where it is difficult to manoeuvre especially if the user has some cognitive disability. To this respect, an important novelty of SHARE-it with respect to similar projects is the use of a holonomic mobile platform, perfectly adaptable to standard indoor environments through special ball-like wheels (Martínez et al., 2005).

Research in AT and mobility aids has also focused in the last years on robotic walkers like VA-PAMAID (Rentscheler et al., 2003), Guido (Rodriguez-Losada et al., 2005), Care-o-bot (Graf et al., 2004), COOL aide (Wasson et al., 2007), ORTW-II (Nejatbakhsh and Kosuge, 2005) or iWalker (Kulyukin et al., 2008). All these systems share the same structure of the robotic wheelchairs (sensors, CPU, control software). Some of these sensor sets usually include handlebar force sensors (Graf et al., 2004; Kulyukin et al., 2008; Wasson et al., 2007) that allow the control system to detect the users' intention in navigation terms. Other sensors included usually focus on obstacle detection. The main focus of the robotic walker research stands for physical impairments while the cognitive aids are usually restricted to navigation support.

Almost all the devices provide help with this obstacle detection and avoidance, the main difference between them lies in the amount of control that the user exerts in the avoidance. Among the different degrees of path planning and guidance services, the user usually selects a destination with the help of a GUI and the systems keep him updated with the current position in the navigation path. Some of the systems permit automated drives like in Graf et al. (2004), while others participate on the idea of shared control (Rentscheler et al., 2003; Wasson et al., 2007), in the sense that react helping the user in danger situations, either by correcting actively the users steering, or passively using the brakes to influence the user forces.

Guido (Rodriguez-Losada et al., 2005) and VA-PAMAID (Rentscheler et al., 2003) both offer the capability of parking itself and coming back to the user at will. Most of these projects remain at prototype stage, lacking experimentation with real disabled users.

\subsection{Environment integration and ambient intelligence}

Ensuring that AT works, not isolated but integrated in a given intelligent ambiance, have brought new research about ambient intelligence (AmI) (Ramos et al., 2008; Augusto and Shapiro, 2007). According to the European IST programme 1999 (ISTAG), AmI focuses on creating intelligent homes to properly help the inhabitants with their ADL. Europe, Japan, Singapore and the USA are paying special attention to this area in 
houses, hospitals and even outdoors. People will live easily in digital environments in which the electronics are sensitive to people's needs, personalised to their requirements, anticipatory of their behaviour and responsive to their presence. Pollack (2005) proposes three goals for intelligent AT addressing cognitive problems:

1 assure that the elder is safe and performing necessary activities

2 assess the cognitive status in the normal living environment

3 compensate the cognitive impairment.

AmI possibilities in environment monitorisation, data gathering and feedback on user activities can contribute to fulfil these goals.

AmI implies three relatively new technologies: ubiquitous computation, ubiquitous communication and intelligent user interfaces. Ubiquitous computation consists of embedding microprocessors, sensors and actuators in everyday objects, so that they acquire some processing capability; it can be perceived how they are used and they can act in an autonomous way. The ubiquitous computing paradigm envisioned by Weiser (1991) is a post-desktop model of human-computer interaction in which information processing has been thoroughly integrated into everyday objects and activities.

One of the pillars of AmI is ubiquitous communications, as information needs to be processed and shared among devices to achieve deliberative decisions. Data processing and distribution and task performing in potentially unstructured dynamic environments is a quite complex problem to solve. Next step in always on communications, new ubiquitous technologies (such as RFID) promise a world of networked and interconnected devices (e.g., fridge, television, vehicle, garage door, etc.) that provide relevant content and information whatever the user's location is. In particular, the convergence (and future scalability) of broadband internet and ubiquitous networks with current mobile services may emerge as the key means for providing communication and monitoring capabilities to users (http://www.itu.int). Multiple technological solutions can be distinguished, presenting differences in aspects like power consumption, range or immunity to electromagnetic interferences. In the concrete case of radiofrequency, the systems exploit technologies widely spread like GSM, Bluetooth, Wi-Fi, Zigbee or RFID, as well as others emerging like ultra wideband technology (http://www.intel.com/go/uwb/) or wireless sensor networks (Marin et al., 2005). Biomedical data collected off patients can be complemented by geographical (subject's location) information, at least as captured by GPS devices (Kamel-Boulos et al., 2009).

Much work has been done on human-computer interfaces (HCI), the main objective of interaction design is maximise the usability of the final product in order to enable its successful use. According to ISO 9241-11 (1998) usability is defined as the "extent to which a product can be used by specified users to achieve specified goals with effectiveness, efficiency and satisfaction in a specified context of use". Especially when designing assistive devices for elderly people, focusing on interaction design that provides good usability is very important, taking into account typical age-related issues, such as sight or movement deficiencies and the impacts resulting from diseases and chronic conditions. In fact, the success of these devices in improving the quality of life highly depends on the degree to which people accept them. The majority of studies that deal with the attitudes of older people toward computer technology indicate that elderly people are receptive to using assistive devices, but that their willingness to do 
so is affected adversely by computer anxiety (Czaja and Lee, 2004; Shneiderman and Plaisant, 2005).

These technologies are related to the key issues to be solved for AmI applications in the AT context:

1 monitoring, as it is necessary to determine the user's localisation at home not only to detect risky situations but also to check his/her ADLs and to determine how much help he/she needs

2 give support to cognitive disabilities, that must be adapted to the user, as too much help might be as undesirable as too little help

3 human factors, as technology must be adapted to the user so that he/she can feel comfortable and in control of his/her ADLs, especially at home

4 decision-making, which is not obvious in this domain, as decisions usually do not follow analytical rules and may need to adapt in time according to user tastes and evolution of conditions.

Telemedicine projects like Doc@Home (http://www.fastuk.org), CHRONIC (http://chronic.cestel.es/) or MobiHealth (http://www.mobihealth.org) could be considered a starting point of AmI applications on the AT context, as they offer monitoring capabilities and ubiquitous data distribution. Confident project (http://212.73.32.174/Fundacion/Europeos/Confident/en/presentation/CF_TOCP.) also offers biometric monitoring and ubiquitous communication infrastructure to communicate elder users with their caregivers. The recently started project Confidence (http://www.confidence-eu.org/) focuses on monitorisation of safety values like fall detection and other unexpected behaviours health related. Other systems take advance of AmI to give cognitive support in mobility and navigation like opportunity knocks (Patterson et al., 2004) that supports real-time route planning or MAPS (Carmien et al., 2005) a prompting system that supports cognitive impaired people on the use of urban transport system.

\section{Aging and mobility}

Transportation and mobility are closely linked to independence, well-being and quality of life. A critical factor in an older person's ability to function independently is mobility, the ability to move without assistance (Guralnik et al., 1993; Espeland et al., 2007). Older people who lose mobility are less likely to remain in the community, have higher rates of morbidity and mortality, have more hospitalisations, and experience a poorer quality of life (Branch and Jette, 1992).

In fact, mobility, in addition to represent one of the most important basic ADL, like defined in the Bartel Index (Mahoney and Barthel, 1965), is directly involved in many basic and instrumental ADL activities like transferring, grooming, toileting, housekeeping, etc. For adults, independent mobility is an important aspect of self-esteem and plays a pivotal role in aging in place (Simpson, 2005). For example, as Simpson suggest, if older people find it increasingly difficult to walk or wheel themselves to the commode, they may do so less often or they may drink less fluid to reduce the frequency of urination. If they become unable to walk or wheel themselves to the commode and help is not routinely available in the home when needed, a move to a more enabling 
environment (e.g., assisted living) may be necessary. Moreover, impaired mobility often results in decreased opportunities to socialise, which leads to social isolation, anxiety and depression (Iezzoni et al., 2001). This is why we focused our attention in mobility and their related activities as crucial issue to guarantee autonomy in elderly and disabled people.

The simultaneous presence of cognitive and mobility impairments has a multiplicative effect, worsening global function more than expected by the sum of the single conditions.

Cognition and mobility heavily affect the capacity of daily planning. For an activity to be effective implies that the person is capable of performing it when he/she wants to or when it is necessary: the possibility of successfully performing daily life connected activities implies the chance of remaining or not in the community.

As a consequence, the capacity of performing ADLs becomes an important indicator of self-dependency or disability, is used as a comprehensive measure in disabled people and can be chosen as a marker of functional status.

It is then mandatory to consider age-related functional status impairment among senior citizens when developing devices to improve disability and to judge their effectiveness in maintaining and improving self-dependency in terms of ADLs.

Figure 1 CARMEN wheelchair (see online version for colours)

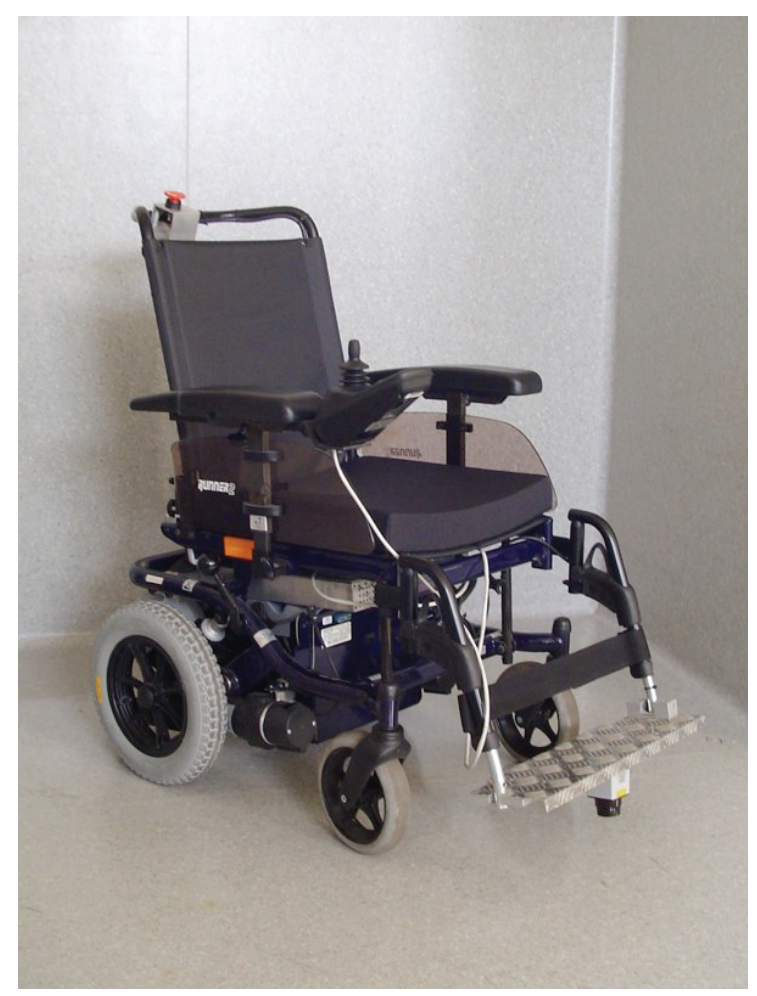


According to these premises, it is quite manifest that independent mobility is critical to individuals of any age. While the needs of many individuals with mobility restrictions can be satisfied with power wheelchairs, some citizens with disabilities find it difficult or impossible to operate a standard power wheelchair. This population includes, but is not limited to, individuals with low vision, visual field neglect, tremors or cognitive deficits. In these cases, a caregiver is required to grant mobility. In order to minimise caregiver support requirements for providing mobility, power wheelchairs can be equipped with an autonomous navigation architecture to assist the user in the control of the wheelchair. The same control architecture can be equipped on top of the $i$-Walker (see Figure 4).

- real environments are hard to predict and highly dynamic (people move around, doors open and close, schedules change, emergencies arise, etc.)

- platforms should be such that they can be easily reused and adapted to already available resources

- it is of key importance to adapt to the user's needs and to avoid providing more support than necessary or to disregard his/her wishes to prevent emotional distress; hence, in most cases control is shared between the user and the autonomous navigation system and the amount of control exerted by the system depends of the user's condition, which may change depending on his/her actual physical/mental condition.

In order to have a wider range of applicability in a real-life environment, and to be able to experiment and evaluate the different platforms, we developed CARMEN (see Figure 1) the Spherik wheelchair (see Figure 3) and the i-Walker (see Figure 4) platforms to be used in our experimentation.

Preliminary results suggest that we are in track to provide the training to inpatients while they are under rehabilitation and then the mobility platforms can go with the user to their preferred environment. We already reported some results on the use of the supported mobility platforms in outdoors scenarios (Barrué et al., 2006; Annicchiarico et al., 2008, 2009).

\subsection{Acceptability and usability}

Finding the right assistive device for each person is not an easy task. Assistive tools have the potential to narrow the gap between an individual's capacity and their environment and, therefore, to make it easier for people to remain in his/her preferred environment. The extent to which these tools can narrow the gap depends on elders' willingness to use it (McCreadie and Tinker, 2005), which in turn depends on several complex factors:

- the needs that people perceive

- safety, may be the most important the perceived usefulness of the AT

- soundness

- degree of autonomy that the assistive tool guarantees to the user.

That is why among the SHARE-it objectives we pursue the idea of personalisation. Personalisation implies a large amount of knowledge about the user's abilities and limitations, his/her environment, his/her clinical information, etc. Personalisation should 
be a sound, safe and easy and adaptive process. Agents have shown to be a solid option to accomplish this aim.

An open research topic is the acceptability of this technology among elders. Senior citizens facing some disabilities need to find this technology easy to learn to use as well as be confident with its usage in their preferred environment. This implies an effort to provide the appropriate infrastructure elsewhere. Also, it should be easy and affordable to adapt these technological solutions to different existing environments.

Usability, within the realm of AT devices, determines how effectively and efficiently AT users with disabilities can function in different contexts and environments (Arthanat et al., 2007). In fact, senior citizens use assistive devices because the functional aspects of those products meet their current needs. One of the key ideas in SHARE-it is to modify the environment, to tailor its attributes, in order to make it more cooperative for the elder and, therefore, compensating for the deficits caused by a poor interaction.

\section{The SHARE-it approach}

\subsection{The SHARE-it architecture}

The overall goal is to create an AmI infrastructure for people with special needs that automatically adapts to their needs and habits. This infrastructure also provides insightful information so that caregivers, doctors and system designers can benefit from the everyday users' experience.

Agents are considered to be autonomous (i.e., independent, not-controllable), reactive (i.e., responding to events), proactive (i.e., initiating actions of their own volition) and social (i.e., communicative). Sometimes a stronger notion is added (beliefs, desires, intentions) realising intention notions for agents. Agents vary in their abilities, e.g., they can be static or mobile, or may or may not be intelligent. Each agent may have its own task and/or role. Agents and MAS are used as a metaphor to model complex distributed processes.

The SHARE-it project offers an innovative solution to improve the quality of living of elderly people with mild cognitive impairments (MCI). The solution is based on the cooperative interaction of several elements: intelligent mobile devices, such as i-Walker (see Section 4.3), Spherik (see Figure 3) or the Rolland wheelchair (Mandel et al., 2005) and AmI components, which serve as tools to doctors and caretakers to provide a better service to the users. Furthermore, the SHARE-it solution works autonomously. Based on user profiles and recommendations from medical actors, it will make decisions to help elders to improve the performance of their daily activities; it will detect emergency situations; and will react by giving an appropriate response. The middleware components both communicate with all actors in the system and perform the autonomous decision-taking actions. In this way, two main functionalities are identified for the middleware:

- Provide efficient and fast interconnection mechanisms for all elements in the SHARE-it solution, giving access not only to information inside SHARE-it, but also extending the communication capabilities to the world outside SHARE-it. 
- Intelligent data gathering and interpretation. This means the middleware is responsible for retrieving all data available from its sources, analysing, filtering and combining them so that only relevant and meaningful pieces of information are stored into its databases. Additionally, the middleware will deliver processed data from their sources to their consumers, i.e., the components needing information to execute their actions. Finally, by combining gathered data, the middleware should infer the status of the system to notice emergency situations and unleash corrective actions.

SHARE-it relies on a MAS for the implementation of the middleware (see Figure 2). The MAS is used for interpretation and intelligent distribution of available information from all sources (the mobile elements and the environment), gathering and distributing it and combining the responses of the different modules to send commands to the actuators. This MAS will also be responsible of the support services - cognitive and mobility related - offered to the users, described in Cortés et al. (2008).

Figure 2 The SHARE-it agent-based middleware architecture

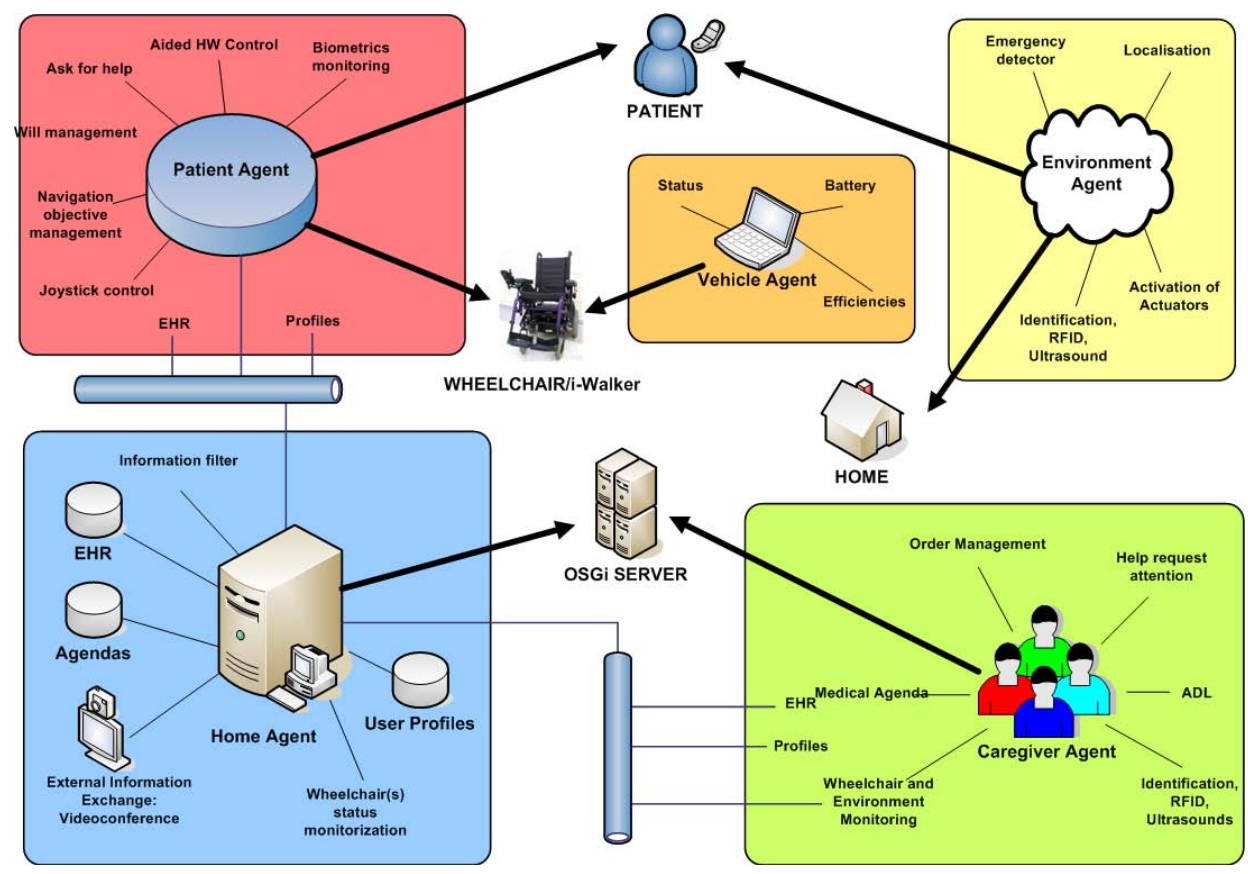

The MAS architecture (see Figure 2) is built up with these elements.

Firstly, we have the patient agents $(p a)$, that runs in PDAs or ultra-mobile PCs. An instantiation of this agent should provide all the available and permitted services to each patient for instance security, mobility, monitoring and help services or new ones that could be added in the future. An important role of $p a$ is to keep the user's agenda. This agenda gives information to other agents to make inferences about user's preferences and habits. Also, it supports the inference of user's immediate goals.

The Vehicle Agent $(v a)$ is allocated in the assistive hardware devices related to the project, be it the different power wheelchairs [Spherik (Martínez et al., 2005) (see Figure 3)] or in the $i$-Walker (Cortés et al., 2008) (see Figure 4). The va supports the 
user in the navigation tasks (e.g., helping to avoid obstacles, etc.) and takes care of the communication with the environment, via the environment agent $(e a)$.

The caregiver agents $(\mathrm{ca})$ is situated in the computers belonging to the caregivers of SHARE-it target population as well as in their individual PDA. The $c a$ is in charge of managing all the user's help request messages, and will notify them to the caregivers, so they can be attended properly. Also, it will notify any detected anomaly in the user's biometric signals and it will generate a request for help, if needed. The ea will run in a network computer connected to all the environment network of sensors (i.e., AmI sensors). Its basic target is to distribute the information from all available sensors to all the agents interested. Finally, we have a home agent $(h a)$ that represents the home entity, which is situated in a home server. Among its objectives are to maintain the monitoring of the users, to manage their daily living activities and their profiles.

Figure 3 The Spherik holonomic mobile platform (see online version for colours)

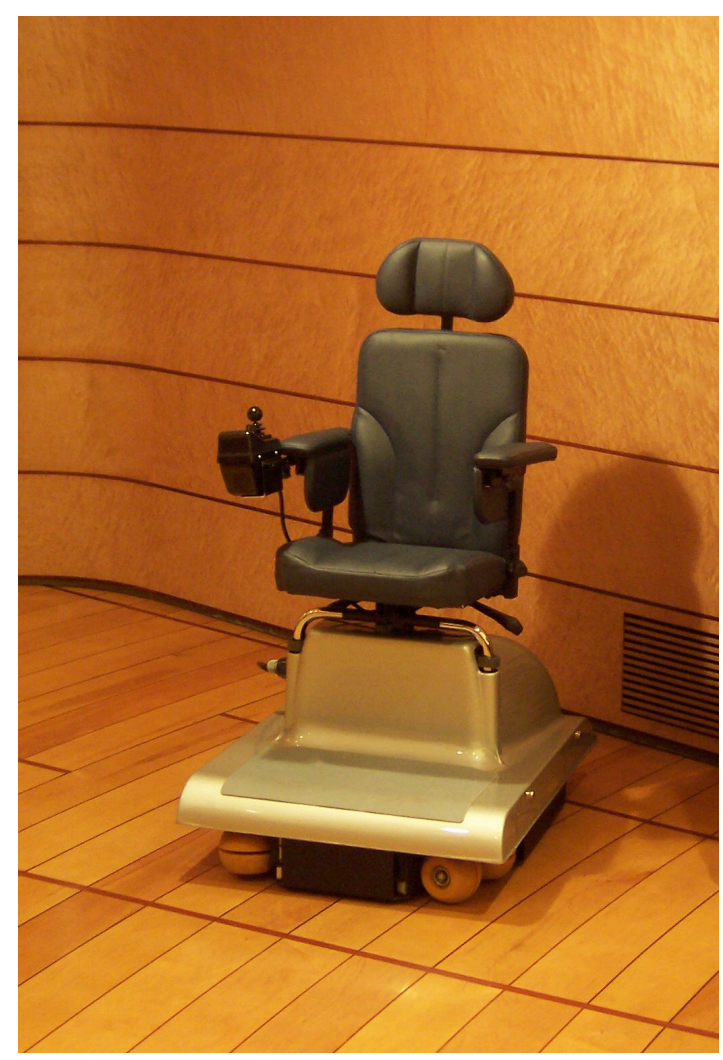

The SHARE-it solution works with medical information regarding actual users. In this scenario, security aspects are of paramount importance, so the system guarantees in every situation that only authorised modules/persons access to sensible data, preserving the privacy of the subjects. 


\subsection{Shared autonomy}

Autonomy for the elderly or people with disabilities does not only rely on mobility terms, but on a set of domains influenced by functioning, activity limitations, participation restrictions and environmental factors. Life areas related to activities and participation are such as learning and applying knowledge, general tasks and demands, communication, mobility, self-care, interpersonal interactions and relationships as well as community and social life. All these domains can be affected by aging or disabilities and are the base of personal autonomy and the satisfactory participation on them reflects on the self well-being. AT are of special interest, as the average age of the population increases fast (Camarinha-Matos and Afasarmanesh, 2001; Pollack, 2005). AT devices can participate in these activities in order to enhance the user's autonomy, gathering all the environmental information and making use of it properly.

Figure 4 The $i$-Walker (see online version for colours)

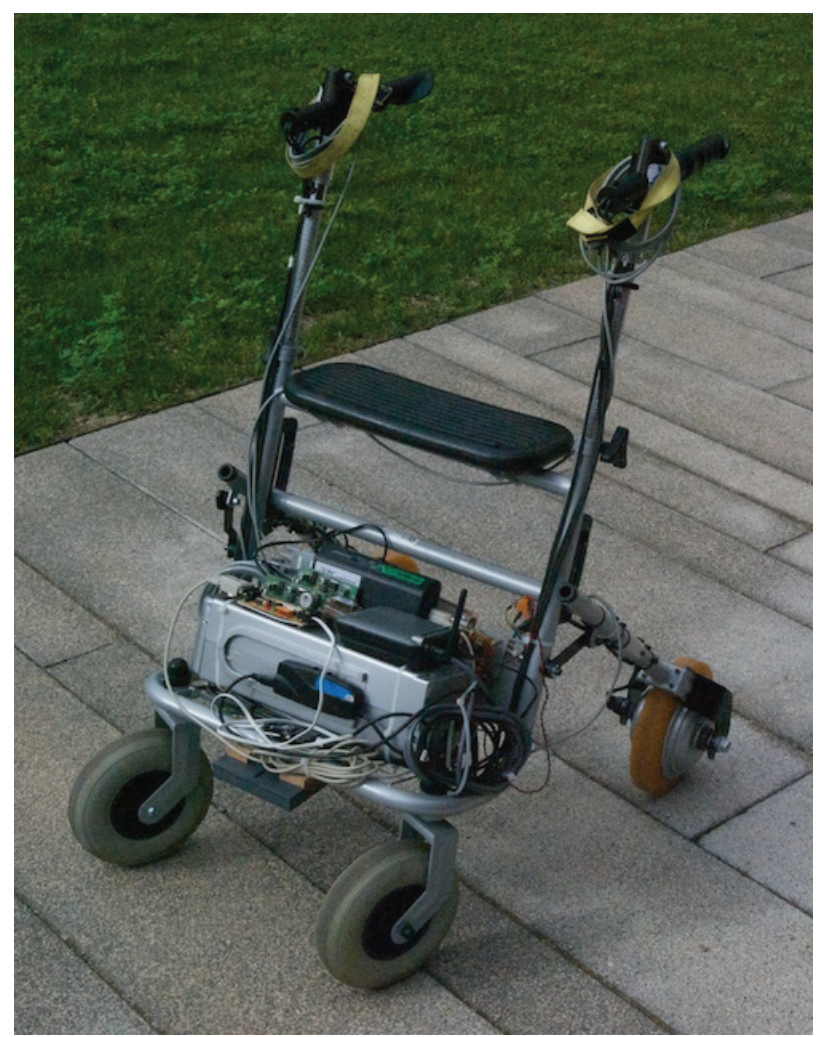

SHARE-it is based on the notion of a shared autonomy between the user and its own agent-based mediator with any information system at hand. From our point of view, existing telematic healthcare systems that provide integrated services to users are not flexible enough to allow real personalisation and, maybe, now it is too expensive to change them.

The shared autonomy concept is scarcely explored in literature and often it is misunderstood as shared control (e.g., Vanhooydonck et al., 2003; Lankenau and Röfer, 
2000). In the personal autonomy and disability context, two different scenarios of the shared autonomy can be elicitaded.

- People presenting mainly physical impairments are able to define their own goals, but due to their restrictions, they usually are not able to execute them, suffering a limitation in their autonomy. In this scenario, the contribution of AT focus on physical devices, mostly mobility hardware, that allow them to reach their objectives. These devices may be controlled by MAS or through an agent supervised shared control if the user's motor capabilities are not severely damaged. In this scenario, user interfaces are very important to detect the user's intention, which is critical to define goals for the wheelchair to be able to assist him/her.

- People presenting mostly cognitive impairments may require a different kind of assistive aids, which may lead even a more relevant role in the sharing of personal autonomy. In this scenario, the user probably does not have very clear goals or is not capable of achieving them because he/she cannot remember how to do them. In these cases, AT may empower and complement their autonomy using agents that offer them a set of services, like reminding what kind of activities they can or should perform at a certain moment of the day or pointing them out how to achieve these activities. The main idea is to offer the users a set of cognitive aids, either rational or memory based, that can ease their daily living.

MAS have both the flexibility and the cognitive capabilities required in order to be able to support the needs of persons with different disability profiles and to complement the autonomy of the people with special needs in an adaptive way through the time. In some cases, the disability is a consequence of a pathology or a trauma, that may improve with some time and rehabilitation. An excess of support or lack of flexibility in the support can make this process more difficult; on the other hand, an assistance adaptive to the daily state of the patient may be helpful in the rehabilitation process.

Some patients may dislike an autonomous navigation system, or choosing among a set of manoeuvres, they may prefer driving by themselves, to feel autonomous and in charge of the situation at all times. An intelligent agent with the necessary knowledge of a user's profile can supervise user's navigation and take part in some driving manoeuvres; in a transparent way, in case the user needs some support (e.g., help crossing doorways, refining turning manoeuvres, help keeping stable cruise navigation, ...).

In order to make this possible, the user's agent must have to have deep knowledge of the user's disability profile and historical data about his/her driving behaviour, merge all this knowledge and translate it in control support and a set of assistive services. All this knowledge and information must be updated dynamically, since the user can progress in either good or bad way or just can have a good/bad day driving-wise. The knowledge learnt by each agent would be shared and distributed among other agents that have users with similar profiles so they can take advantage of the experiences traced by the first one. Agent's responsibility grows with the measure of his active intervention in the user's autonomy is exerted. This means a heavier charge of obligations regarding safety and soundness in the undertaken actions.

The main open issues in shared autonomy that we identify as crucial are those related with the assessment of disruptive and/or unexpected events where the system has to act proactively but also in a safe and sound way for the user. 


\subsection{The i-Walker platform}

Among the mobility platforms developed in the SHARE-it project we will present, in this section, the $i$-Walker as an example of the possibilities and generality of the technology. This platform has been developed in several variants for different kinds experimentation, with minimal development effort, based on existing HW-components as much as possible. The idea is to reach a much larger share of the aged population with this platform, compared with power wheelchairs, while porting as much of the existing AT developed for CARMEN, Rolland and Spherik as possible. The ported technology ranges from hardware components (laser scanners, odometry, sensors for monitoring, integration with the environment, multimodal communication) to the software to control it.

Previous experience with assistive walkers [e.g., Thrun et al.'s seminal work (Glover et al., 2003; Rodriguez-Losada et al., 2005)] shows that giving navigation directions to aged persons is a definite success; the ability to safely reach a desired goal (e.g., the cafeteria, a friends room in a building), and come back (!), overcomes uncertainty barriers and increases mobility and the motivation for exercise through walking. However, after discussion with medical experts about safety issues, we are not convinced that a walking assistant should actively move [as, e.g., the SmartWalker Guido in Rodriguez-Losada et al. (2005)]. Among of the SHARE-it objectives is to build different $i$-Walker workbench platforms, oriented to demonstrate their feasibility and gain the confidence to support the specific disabilities (Cortés et al., 2003). Two inspiring works in this line for intelligent pedestrian aids are Glover et al. (2003) and Wasson et al. $(2001,2003)$. They proposed intelligent robotics agents to support elders mobility but the main difference in our approach is the explicit use of intelligent software agents to support decision-making and to help in the interfacing with the user. More modern lines of research in the field of pedestrian mobility are represented by Cheng et al. (2008) and Kulyukin et al. (2008). The first is more oriented to avoid the fall risk and the second culminates a line of walkers developed at CMU. Kulyukin et al.'s $i$-Walker is intended to guide elders in a small world and it is not prepared to avoid unexpected obstacles in that environment. Other walkers incorporate robotic aids that are prepared to help users to change their position as from sit-to-stand (Chugo et al., 2008). Standing up motion is one of the most serious and important operations in daily life for elderly person who does not have enough physical strength. Thus the $i$-Walker, like Rolland, allows:

1 safely avoid collision, circumnavigate obstacles, guide through doors, etc.

2 interact with the user via a multimodal interface and linguistic dialogue

3 direct the user to selected goals, giving route directions.

Apart from the multimodal (in particular speech) interface, we are experimenting with moderate brake on the $i$-Walker wheels to gain the experience on how to guide the user by allowing $\mathrm{s} / \mathrm{he}$ sharing with the computer the driving and steering actions, respectively. Figure 5 shows a top view of an experimental $i$-Walker with two front castor wheels and two rear wheels that can be moderately braked. If the user can guide the $i$-Walker this wheels configuration will allow a complete differential steering, but if the user cannot participate in the steering actions then a restricted differential steering is achieved. In any case, Figure 5 illustrates how the action of computer-controlled braking can change 
the $i$-Walker trajectory and, therefore, guide the user around obstacles (1) and in the desired direction (3), but the user is expected to walk actively her/himself. The choice of having computer-controlled brake actions add-on to the walker another interesting optional feature: the ability to program specific additional effort while pushing the walker by medical prescription. And, indeed, feedback the user about the accumulated daily distance seems to be an additional motivation for exercise. In addition, following an idea of Thrun et al., we shall endeavour to let the $i$-Walker park itself automatically, and come back to the seat on demand by remote control (this avoids unsafe staggering by the user from the parking position to a remote seat and back). The choice of the DC motors for the driving wheels of the walker will have to take into account: freewheeling when the user goes in the right directions; computer-controlled brake actions to guide the user when his/her orders are wrong; and finally, the need of computer control of the wheels speed to guide the $i$-Walker to a park place.

Figure 5 i-Walker braking control, forces interaction

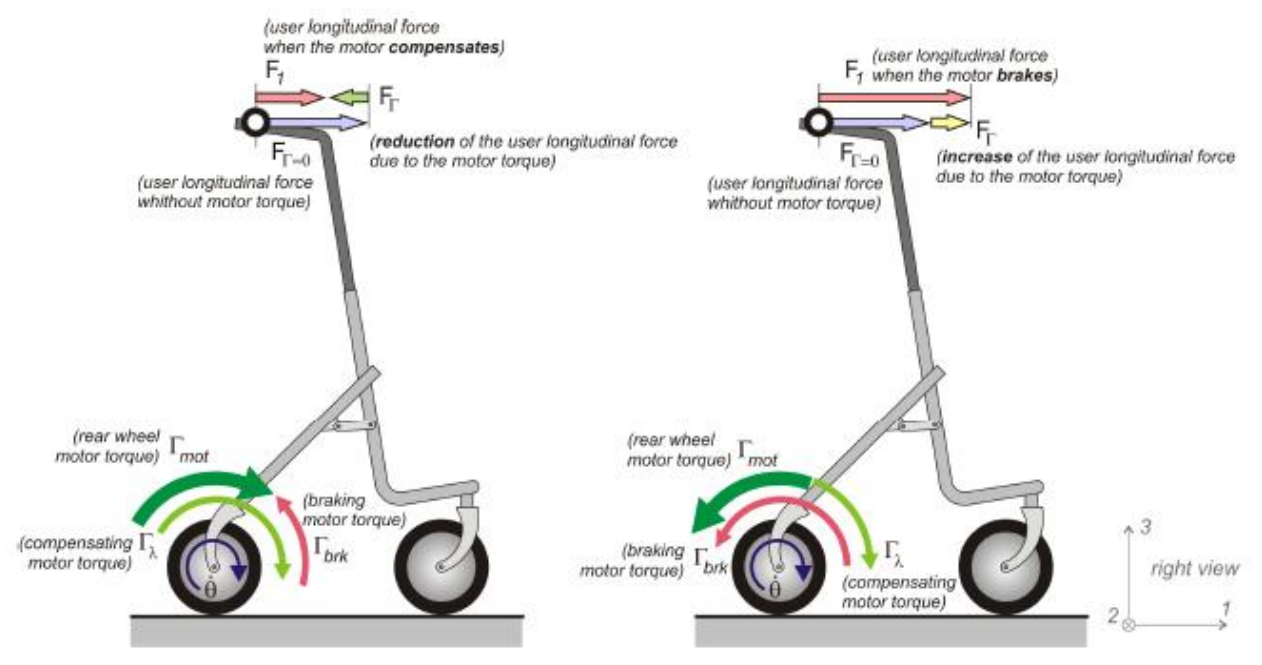

\section{4 i-Walker control concept}

The i-Walker has been designed to be passive, cooperative and submissive (see Figure 5).

- Passive, because it can only adjust the facing direction of its front wheel, i.e., it can steer. However, it has no forward drive motors and so relies on the user for motive force. This allows the walker to move at the user's pace and provides for the user's feeling of control.

- Cooperative, because it attempts to infer the user's path and uses this inference to decide how to avoid any obstacles in the user's path.

- Submissive, because it monitors the users to see if they are resisting the actions (steering/braking) selected by the walker. If they are, the movements are adjusted. This cycle continues until the user agrees with the motion (i.e., does not resist it) 
or manually overrides it. This interaction forms the basis of the feedback loop between user and agent.

The manual brakes have also been replaced with an automated braking system. The $i$-Walker can sense the user's steering input via sensors in the handles that detect the difference in force on the two handles.

- pushing with more force on one handle (left or right), the walker will turn in the opposite direction

- applying of equal force on both handles will move the walker straight forward or backward (which direction can be determined by the $i$-Walker wheel encoders).

One of the main objectives of SHARE-it is helping the users in orienting them when handling the $i$-Walker in a known environment. The orientation service is provided by an agency already fully described in Barrué et al. $(2004,2006)$ for a power wheelchair.

The user will receive help from a screen, but the innovative idea will be steering by moderate braking, for helping in navigation. The main idea is to help target population while driving the $i$-Walker in normal situations like: uphill, downhill, turning left/right and/or standing still, standing up, etc.

\section{Collaborative control}

The SHARE-it approach relies on giving the users just the amount of assistance that they need in order to drive their mobility platforms. Thus, we avoid loss of residual capabilities and enhance the feeling of autonomy of assisted people. In this section, we explain the case of a power wheelchair. Navigation can be defined as the act of reaching a destination in a safe way. From this definition, goals in this activity can roughly be defined as physical locations which, at hardware level, are translated into commands to the motors that drive the wheelchair towards those positions in a safe way. Commands sent to the wheelchair motors have a component of human input and machine orders too. Specifically, humans provide commands to the chair via suitable interfaces (joystick, touch pads, buttons, trackers, voice, ...), whereas the machine uses algorithms, available models of the environment and hardware and the readings of the onboard sensors to calculate a feasible route and decompose it into motor commands. The main problem of this approach is that most times the system must reconcile two potentially different goals, like trying to keep as far as possible from obstacles and moving in a particular direction at the same time. For this reason, we have chosen to work with reactive navigation schemes, as they are particularly interesting because they may deal with several sensors and goals in a simple way. Hence, we use a potential field approach (PFA) (Khatib, 1986) to combine human and wheelchair commands and goals (Sánchez-Tato et al., 2007). It is necessary to note that this combination, though, is not be completely reactive, as humans tend to have a deliberative agenda that is propagated down to joystick commands. Human influence on a reactive layer may be of help to gain global efficiency via prediction and to avoid local traps. If the user presents a cognitive disability that prevents him/her to make deliberative plans, a deliberative layer could be also added to the mobility platform in a hybrid way. To this respect, projects and labs like COACH, PlaceLab, Tigerplace or LACE already focus on statistically modelling specific activities from a given ADL into isolated actions, that can be predicted, detected 
and analysed. Markov models are usually employed to obtain transition probabilities from each state to the next and, given the appropriate circumstances, either fill the gaps or trigger alarms. In any case, activities related with navigation could be decomposed into a chain of places to visit and, hence, a set of partial goals that can be handled by the proposed approach.

PFA basically relies on modelling obstacles as repulsors and goals as attractors to create a vector field that returns a motion vector at each point. In order to deal with common indoor, guided navigation, we have modified PFAs to keep obstacles at the sides of the wheelchair at the same distance by adding three forces [Figure 6(a)]: $f_{C F \text { paral }}$ tries to move the mobility platform parallel to a wall. $f_{C F \text { dist }}$ takes into account the wall on the other side, should there be any. Finally, $f_{C F a v o i d}$ avoids obstacles that may appear in the way of the chair. Let $k_{C F \text { paral }}, k_{C F d i s t}$ and $k_{C F a v o i d}$ be three factors that weigh $f_{W F \text { paral }}, f_{W F \text { dist }}$ and $f_{W F a v o i d}$, respectively. If $d_{C}$ tends to the the distance to the obstacle, $v_{r R}$ is calculated as:

$$
\begin{aligned}
v_{r R} & =f_{\text {CF paral }}+f_{C F \text { dist }}+f_{\text {CFavoid }} \\
& =k_{\text {CF paral }} \cdot \alpha_{W} \\
& +k_{C F \text { dist }} \cdot\left(d_{C}-d_{W}\right) \\
& +k_{\text {CFavoid }} \cdot\left(d_{C F S E C}-d_{F}\right)
\end{aligned}
$$

$\alpha_{W}$ being the angle to the nearest right wall; $d_{W}$ being the current distance to the right wall; and $d_{F}$ being the distance to obstacles in front of the mobility platform. $d_{C F S E C}$ is a threshold distance to consider that an obstacle is too close to the mobility platform. It can be noted that in absence of walls, this equation tends to be equal to a purely reactive PFA.

Figure 6 PFA forces for: (a) corridor path and (b) door crossing

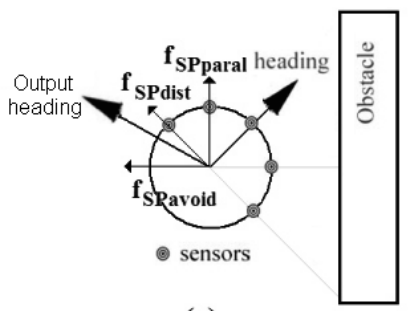

(a)

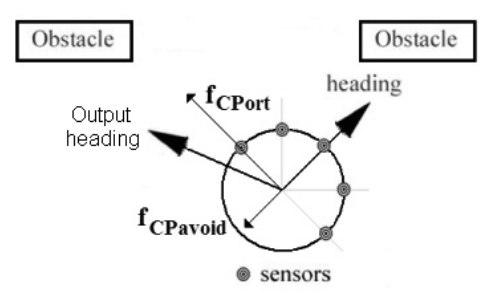

(b)

In order to also avoid oscillations and incapacity to cross doors, when a door is detected in front of the wheelchair (as a wall opening), a door crossing behaviour, also based on PFA, is triggered to move through. This behaviour is based on two forces [Figure 6(b)]: $f_{D C o r t}$ allows the mobility platform to stay orthogonal to the doorframe; and $f_{D C a v o i d}$ avoids the doorframe, preventing the mobility platform from colliding with it. Let $k_{D C o r t}$ be a constant that weighs $f_{D C o r t}$ and $k_{D \text { Cavoid }}$ be another constant that weighs $f_{D \text { Cavoid }}$ s. Let also be $d_{L}$ and $d_{R}$ the distance to the left and right part of the door, respectively. If $d_{F}$ is the distance to obstacles in front of the mobility platform, $v_{r R}$ is:

$$
\begin{aligned}
v_{r R} & =f_{\text {DCort }}+f_{\text {DCavoid }} \\
& +k_{\text {DCort }} \cdot\left(d_{L}-d_{R}\right) \\
& +k_{\text {DCavoid }} \cdot\left(d_{D C S E C}-d_{F}\right)
\end{aligned}
$$


being $d_{D F S E C}$ a threshold distance to consider that an obstacle is close enough to the mobility platform to be of interest.

The output of the mobility platform motion is a motion vector that may be combined with the human command vector. This approach has the side effect of avoiding local minima, as humans provide an uncorrelated force to the vector combination. Basically, the user provides a direction that is added as another vector in the potential field at each position (Figure 7). The key to achieve collaborative motion that preserves efficiency and safety and, yet, allows the user to be in control at all times is to properly determine how to weight the human and mobility platform vectors when adding them. In this work, weights are proportional to the efficiencies of human and mobility platform at each time instant. Usually, mobility platforms will be more precise, whereas humans will be more versatile. If both perform equally well, the global command will be the average of them both. This should not be a problem in control perception from the user's point of view, as similar efficiencies at local level correspond to similar motion commands also. The local character of efficiencies is forced by the purely reactive nature of the approach. Consequently, it is necessary to determine which factors can be used to measure efficiency in a punctual way.

Figure 7 Local efficiency factors for human and mobility platform

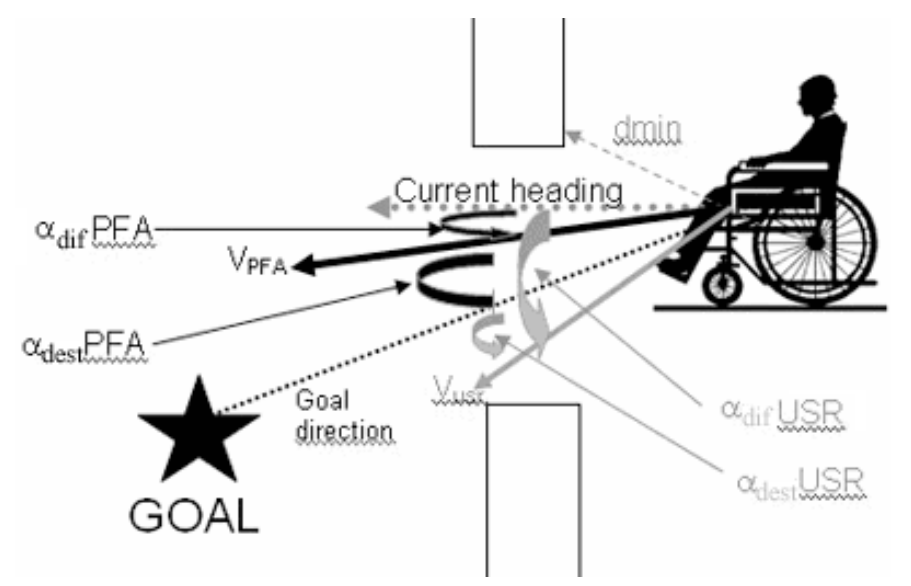

The reactive behaviour provides the rotational $\left(v_{r R}\right)$ and translational $\left(v_{t R}\right)$ velocities the wheelchair supplies as its own motion velocities $\left(v_{r H}\right.$ and $\left.v_{t H}\right)$. Shared motion commands (rotational velocity, $v_{r S}$, and translational velocity, $v_{t S}$ ) are defined by:

$$
\begin{aligned}
& v_{r S}=\eta_{R} \cdot v_{r R}+\cdot \eta_{H} \cdot v_{r H} \\
& v_{t S}=\eta_{R} \cdot v_{t R}+\cdot \eta_{H} \cdot v_{t H}
\end{aligned}
$$

where $\eta_{R}$ is the efficiency of mobility platform motion commands and $\eta_{H}$ is the efficiency of human motion commands. Both mobility platform commands and human output are added as weighted vectors (Figure 7), so that humans receive more control as a reward for a better efficiency. The shared motion command efficiency is defined as $\eta_{S}$. Efficiencies range from 0 to 1 , being 1 the maximum efficiency. It must be noted that $\eta_{S}$ is not equal to $\eta_{R}$ nor equal to $\eta_{H}$. Since shared commands linearly combine both mobility platform and human ones, $\eta_{S}$ will tend to average $\eta_{R}$ and $\eta_{H}$. 
It is fairly easy to weight these factors using biometrics or medical constraints as well (Fernandez-Espejo et al., 2007) but, in this case, only reactive factors are taken into account.

As combination is performed at reactive level, efficiencies $(\eta)$ should only be evaluated in terms of factors having an immediate effect on navigation. Consequently, three factors are taken into account: smoothness $\left(\eta_{s f}\right)$, directiveness $\left(\eta_{t l}\right)$ and safety $\left(\eta_{s c}\right)$, each of them ranging from 0 to 1 . Smoothness reflects how sharp direction changes are undesirable for driving. Safety reflects that it is better to keep away from obstacles. Directiveness tries to reflect that moving ahead to the goal in a straight way leads to shorter paths.

Smoothness $\left(\eta_{s f}\right)$ is locally evaluated as the angle between the current direction of the mobility platform and the provided motion vector. Smoothness is reflected on the emergent curvature of the mobile platform, where curvature is a measure of how much a curve bends at each point. This factor is included because mobiles may be non-holonomic, meaning that they cannot change directions abruptly due to their kinematics. Consequently, it is better to change heading as less as possible to avoid slippage and oscillations. If $C_{s f}$ is a constant and $\alpha_{d i f}$ is the angle difference between the current direction and the command vector, $\eta_{s f}$ will be:

$$
\eta_{s f}=e^{-C_{s f} \cdot\left|\alpha_{d i f}\right|}
$$

Directiveness $\left(\eta_{t l}\right)$ is locally measured in terms of the angle formed by the mobility platform heading and the direction towards the next partial goal provided by the global planner. Obviously, the shortest way to reach that goal is to go straightly towards it, consequently making the angle zero. Obviously, any obstacle in the way would prevent that movement, but this is contemplated by another factor. Let $C_{t l}$ be a constant and $\alpha_{\text {dest }}$ the angle between the mobility platform heading and the direction towards the next partial goal. Hence, $\eta_{t l}$ is calculated as:

$$
\eta_{t l}=e^{-C_{t l} \cdot\left|\alpha_{d e s t}-\alpha_{d i f}\right|}
$$

The third factor, safety $\left(\eta_{s c}\right)$, is evaluated in terms of the distances to the closest obstacles at each instant with respect to the heading direction. The closer the wheelchair gets to obstacles, the less safe the trajectory is. Assuming that $C_{s c}$ is a constant and that $\alpha_{\min }$ is the angle difference between the current direction and the direction of the closest obstacle, $\eta_{s c}$ will be:

$$
\eta_{s c}=1-e^{-C_{s c} \cdot\left|\alpha_{m i n}-\alpha_{d i f}\right|}
$$

Finally, efficiency is obtained through the combination of the three aforementioned factors:

$$
\eta=\frac{\eta_{s f}+\eta_{t l}+\eta_{s c}}{3}
$$

All mentioned factors are reflected in Figure 7 both for human and mobility platform. It is necessary to note that it is not necessarily advisable to achieve a local efficiency equal to 1. First, some efficiency factors are opposite in presence of obstacles, like keeping far from obstacles and trying to turn as little as possible. Also, the layout of the environment may make it necessary not to head to the goal at all times, but, being this a global consideration, the local efficiency may not contemplate this fact. Finally, in order 
to move through narrow places like doors, security efficiency might be low sometimes. Equation (8) is basically used to decide who makes the smarter move at each point, human or wheelchair, so that they get awarded with more control, but not neglected from the emergent behaviour nevertheless: if human efficiency is bigger, motion mostly obeys the driver, whereas if it is small, the wheelchair tends to move on its own.

The proposed approach has several advantages:

1 it tends to preserve curvature and to grant safety, as most PFA-based algorithms

2 humans are in control all the time and they do not perceive sharp control swaps

3 humans provide deliberation and avoid local traps.

Furthermore, the proposed reactive scheme is fully compatible with higher level navigation layers to achieve a hybrid navigation system if necessary. However, even though efficiencies obtained from trials based on this approach are over $70 \%$ in all cases and resulting tracks are consistent with the users' goals, psychological factors also need to be taken into account to obtain a cooperation as seamless as possible between user and wheelchair.

\subsection{Adaptation to the user}

Results obtained in different trials with volunteering in-patients with different degrees of disability have provided intriguing results. Even though, as mentioned, all in-patients ranked above $70 \%$ in efficiency, we detected that some in-patients presenting both better cognitive and physical abilities than the others presented lower efficiencies when compared to them. We also checked that, in general, in-patients presenting poorer cognitive capabilities had a higher efficiency than the rest. In our tests, we used a comprehensive geriatric approach (Nikolaus et al., 1999) and, in particular, we mainly evaluated the cognitive impairment through a neuropsychological battery (Carlesimo et al., 1996). After considering these results, we checked the difference between human input and machine commands. The angle between both vectors, that we call disagreement, provides an idea about how well human and machine cooperate with each other. We wanted to check if a high disagreement caused a loss of efficiency, meaning that users are not really cooperating with the chair, but rather fighting it. As expected, we checked that most outliers, in terms of efficiency, corresponded to persons presenting a high disagreement. These outliers corresponded mostly to two different cases:

1 persons with good cognitive abilities and low physical skills

2 persons with good cognitive and physical skills.

In both cases, users had their own tendencies when driving, that did not necessarily match the machine outputs. In fact, their responses were not necessarily bad, only less efficient than the machine from a local point of view.

In order to provide a better adaptation to the user and, hence, reduce disagreement, it is interesting to check if we can learn how a given inpatient controlled the chair from his/her trace. As the presented work has been performed at reactive level all through the experiments, it was decided to also capture the reactive nature of their driving behaviour, meaning that we search for a duplex of wheelchair input readings (range sensors) and output command (joystick). This pair characterises what the user does at every given situation. In order to capture these pairs, we have used case-based reasoning (CBR). 
A CBR system cycle to solve a new problem consists of four steps (Aamodt and Plaza, 1994):

1 retrieve the most similar stored case or cases to the new current case

2 adapt its solution to the new current case $^{1}$

3 evaluate the results of the proposed solution

4 learn from the new experience.

CBR has been used in mobility platform navigation before, but typically for high level planning rather than to accomplish reactive behaviours (Branting and Aha, 1995; Likhachev et al., 2002; Ram et al., 1993; Urdiales et al., 2006). In our case, each time a new input instance is detected, the CBR captures a new case, coupling this instance with the user's joystick output and using the local efficiencies (directiveness, smoothness and safety) to rank how good the case is. The input instance describes the relative position of obstacles with respect to the wheelchair and the goal. In this case, these distances are split into bins corresponding to danger/near/medium/far/no influence relative distances between chair and obstacles in order to keep a bounded number of cases learnt, as proposed in Urdiales et al. (2006).

Then, a clustering algorithm is used to turn this case set into a casebase. This clustering process had several goals. First, it avoids too large casebases, as similar cases are averaged into a prototype. Second, cases associated to similar sensor readings but different joystick outputs tend to provoke oscillations. These cases, too, are averaged into the same prototype. Finally, the clustering process filters punctual cases due to errors and remove them from the casebase.

In standalone mode, an input vector (sensor readings plus goal) is fed to the CBR server. The CBR server access the casebase and looks for the most similar case available and returns it to the client. This case includes also the joystick readings, which is what the emulator assumes that the user would have done in the input situation. In order to determine the likeness between cases, we used a Tanimoto distance (Rogers and Tanimoto, 1960). The main difference between this type of distance and a Euclidean one is that it weights the global shape of the instance rather than isolated similarities alone. Hence, matching laser readings presenting low distances are related to similar surrounding geometries because the global shape of the case is weighted.

After the specifics of a given in-patient have been captured into a casebase, the CBR system can be used to replace the PFA, so that the wheelchair drives like the user and, hence, disagreement is reduced. It is interesting to note that different users generate different casebases and, hence, different ways of driving. In our experiments, we duplicate how the user drives with collaborative control, because users might not be capable of driving on their own. However, other experiments may be based on traces captured from persons driving on their own.

Figure 8 presents the proposed collaborative control scheme after CBR learning is included in the architecture. It can be observed that the emerging motor commands are still the linear combination of human and wheelchair commands, which are directly coupled with the sensory input (either human or mechanical) in a purely reactive way. These commands are weighted by their average efficiencies, so that low human efficiencies imply higher help from the machine. The main difference when we include CBR learning is that cases related to specific situations are stored in terms of what 
an specific person would do given that situation. Reflexes are implicit in people, so the casebase basically stores how the person drives the wheelchair. Through use, the casebase outputs grow more and more similar to the user's commands. However, if a person drives the wheelchair badly, those cases would also be learnt by the chair. In order to avoid this, we learn cases after they are modulated by either the PFA algorithm or an efficient human command, coming from the PFA block or an already existing case, respectively. Whenever an input situation is detected, the system asks the CBR for the closest case available. If there is none, we use a mix of PFA and human commands as output and learn the corresponding case. If there is any, we use a combination of human and CBR commands as output and, if necessary, learn this combination as well, so that control tends to be progressively more tuned with the user's guidance.

Figure 8 Proposed collaborative control scheme

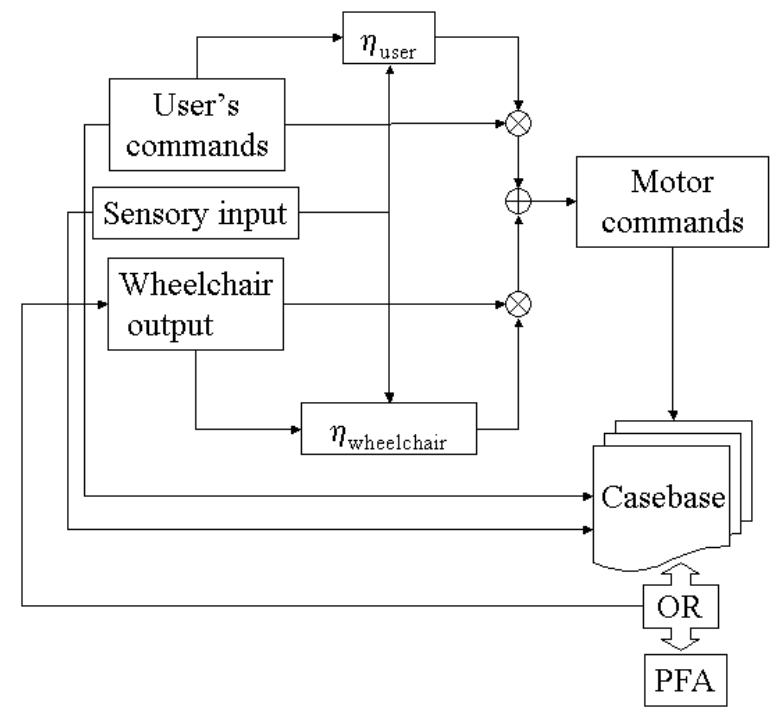

In work to come, SHARE-it aims at running a CBR learning system along with the collaborative control scheme all the time, so that the casebase grows more similar to the user's guidance in time and, hence, driving is tuned to the user's preferences. If the user's efficiency is very low in some specific situations, the system will learn more from PFA than from the user. Otherwise, it will grow progressively more and more similar to the user. Hence, disagreement will be reduced and wheelchairs and persons will cooperate in a harmonic way.

\section{Results}

One of the main contributions of this work is in studying the real use of AT by real users. We carried out a first experimental evaluation of an agent-based robotic control system via a control experiment measuring the performance of a group of users inpatients at the FSL presenting different pathologies - depending on how much control they exert over their wheelchairs, following the methodology explained in Section 5. 
Results are correlated with users' pathologies to extract conclusions about the possible benefits of agent-based assistive navigation and how existing approaches could be improved through adaptation to the user (Urdiales et al., 2006, 2009).

A more exhaustive set of tests with Rolland, CARMEN and $i$-Walker have been performed with aid of 21 volunteers, in the autumn of 2009. This experimentation phase was based on the integration of the SHARE-it mobility platforms in a space called Casa Agevole (Vescovo, 2005). This house is a metaphor for a real ambient intelligent space that has been specially designed for elders to live in without architectonic barriers. Casa Agevole is a fully controlled and instrumented house in $60 \mathrm{~m}^{2}$ (see Figure 9). As a part of the experimental design, it was decided not to perform any architectonical modification to its original setting. In this space, future FSL inpatients will be trying the technology before they return to their homes. The basic element of these experiments was the notion of scenario. A scenario is an example narrative description of typical (repetitive) interactions of users with the system.

Figure 9 The Casa Agevole

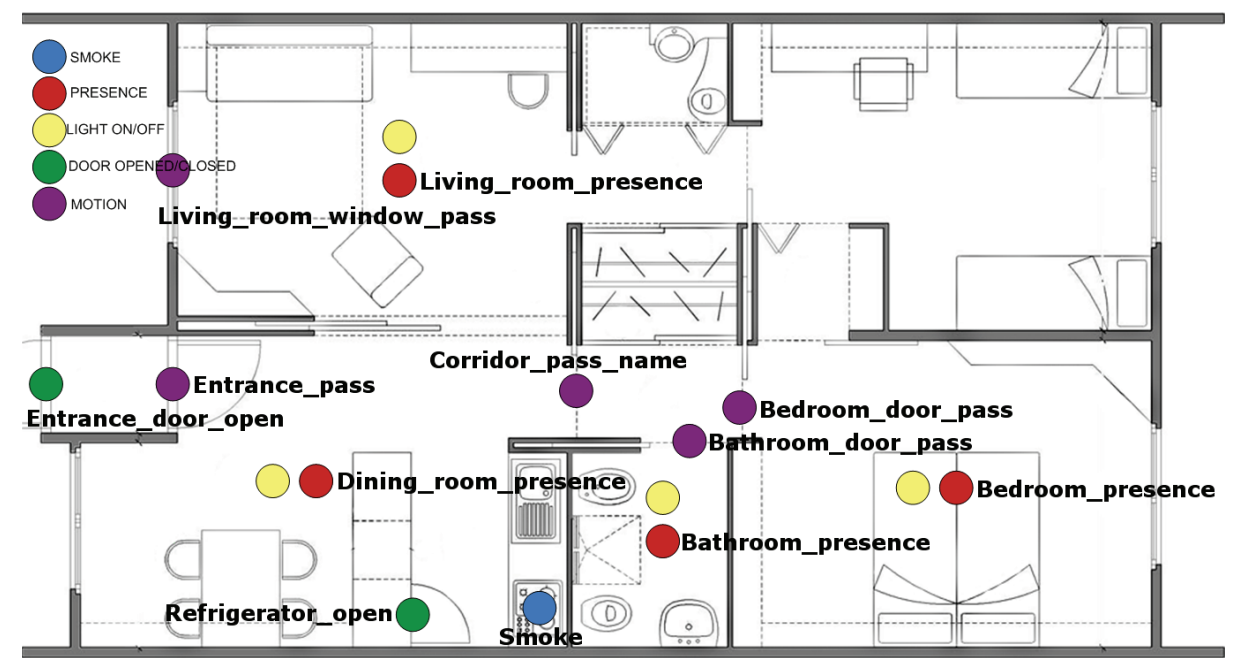

A scenario has this form:

"Alberto uses an $i$-Walker, he does not have a real deficit of the gait, but as many cognitively impaired patients, he feels much more confident if he can rely on a walking aid. He usually wakes up at 9 AM. After having his breakfast, Alberto has to take some pills, as part of his drug therapy, but since he suffers from memory impairment, he cannot always remember that. The same situation repeats three times: after breakfast, in the middle of the afternoon and at dinner. Early, in the morning, before leaving home Alberto's daughter subdivides the proper amount of the different drugs that have to be taken during the day in three boxes, one for each medication episode, different in colour and shape. This requires minimum effort from the caregiver and the support of the system will allow Alberto to manage his therapy alone. At 10:00 AM, the screen mounted on the $i$-Walker shows the system reminder; it consists of both a visual suggestion and a sound alarm, inviting Alberto to take his pills. The system then asks Alberto to confirm if he has taken his drugs. If the system does not receive it, it will send an alarm message (via SMS) to the caregiver. The same procedure will be repeated every time Alberto has to take his therapy." 
Each scenario includes:

1 Target group: A description of the typical user for the scenario.

2 Purpose of the scenario: Describes which aspects of the system and its interactions with the user are focused by the scenario.

3 ADL scenario: The scenario provided by the application domain experts. It describes the ADL, without the use of SHARE-it technology. Therefore, this scenario is the as-is scenario.

4 Scenario text: The narrative that describes an example of the user's interaction with the system.

5 Roles of the agents: Describes the interactions between the different agents and the technology they represent. The main agent types interacting in these scenarios were described in Section 4.1.

The feelings reported by the volunteers after using the $i$-Walker, in the scenario 'Alberto takes his drugs', confirmed the usefulness and usability of the forces compensation help strategies (see Section 4.3) as well as the integration of the offered services.

For most volunteers, using Rolland or CARMEN, it was immediately clear that they would not be able to drive the mobility platform without assistance in a narrow environment such as Casa Agevole. The automatic correction of speed and steering for obstacle avoidance and bypassing can be considered a success. Volunteers positively valued CARMEN. This wheelchair looks almost like any common power wheelchair and it is controlled in the same way, so that user's reluctance to deal with new technology was more or less the same as a novel power wheelchair driver. They became steadily confident on the wheelchair control strategies (see Section 5.1), as they acknowledged that navigation was safe. CARMEN adapts the amount of assistance given to the driver depending on his/her needs and caregivers, doctors and users have positively perceived this. However, users with a better cognitive profile are more reluctant to receive help even when they need it if they realise that the outcome of its commands is not what they expected. The navigation layer has been modified for those cases, and the new tries to mimic the driver's behaviour via CBR learning.

The complete clinical results are still under analysis and validation.

\section{Conclusions}

Assistive tools designed for an elder must, in our conception, be conceived of as part of a larger system of existing products and environments that serve elders and others living/acting in the same environment. As doctors reported from the beginning, there is no such thing as a standard user or a disability unique profile. Hence, there is no single solution to cover all possible functional disability profiles. Therefore, the major problem is to identify the possible ways of interaction between the user and the tools. This implies to assure both usability and accessibility. We observed elders using computers and assistive tools to identify specific barriers to successful use, and then sought free and/or low-cost accommodations to the interface to make computers and assistive tools more easily usable by elders. Though, there is no, in the best of our knowledge, a clear way to do this and assure that all the possibilities of interaction are all covered. 
We are aware that people age differently, and AT devices must get smarter as a person's cognitive and/or motor functioning declines. Our vision is to produce the next generation of AT that enables the target population to increase their autonomy, trying to cope with the cognitive decline in the adequate measure, in their preferred environment, far beyond performance levels of today. The two key elements of this new generation are: personalisation and agent-based support. We emphasise the autonomy of the elderly as a primary goal of our research. The paper provides an overview of the SHARE-it hardware and software platforms making up the agent-based control system.

$i$-Walker is in itself a stepping stone in the process of enhancing assistive tools for the elders. There is a strong case for the use of the $i$-Walker inside the frame depicted by SHARE-it and, therefore, for the use of intelligent agents to support mobility and communication in senior citizens. Moreover, there is a clear evolutionary pathway that will take us from current AT to more widespread AmI where MAS will be kernel for interaction and support for decision-making. The ultimate goal of the interaction between robotics, agent systems and the user is to enhance autonomy and upgrade the quality and complexity of services offered.

We firmly believe that the SHARE-it approach and architecture will contribute to the news trends among Gerontechnology researchers and other stakeholders that advocates for shift from institutional to preferred environment (home-based) care. In this new scenario, the agent-based technologies and solutions, we are proving may positively benefit and help elders, with mind cognitive problems, to age-in-place. In our view, the user should only be assisted according to his/her profile: not more, not less.

\section{Acknowledgements}

The authors would like to acknowledge the support from the EC funded project 'SHARE-it: Supported human autonomy for recovery and enhancement of cognitive and motor abilities using information technologies' (FP6-IST-045088). The views expressed in this paper are not necessarily those of the SHARE-it consortium. The project was running from 1 January 2007 until 31 December 2009.

\section{References}

Aamodt, A. and Plaza, E. (1994) 'Case-based reasoning: foundational issues, methodological variations and system approaches', AI Communications, Vol. 7, No. 1, pp.39-59.

An Information Capture and Processing Environment for Chronic Patients in the Information Society (Chronic), available at http://cordis.europa.eu/fetch?ACTION=D\&CALLER=PROJ_IST\&RCN=53659.

Annicchiarico, R., Barrué, C., Benedico, T., Campana, F., Cortés, U. and Martínez-Velasco, A. (2008) 'The i-Walker: an intelligent pedestrian mobility aid', in M. Ghallab, C.D. Spyropoulos, N. Fakotakis and N.M. Avouris (Eds.): ECAI, Frontiers in Artificial Intelligence and Applications, Vol. 178, pp.708-712, IOS Press.

Arthanat, S., Bauer, S.M., Lenker, J.A., Nochajski, S.M. and Wu, Y.W. (2007) 'Conceptualization and measurement of assistive technology usability', Disability and Rehabilitation: Assistive Technology, Vol. 2, No. 4, pp.235-248.

Augusto, J.C. and Shapiro, D. (Eds.) (2007) Advances in Ambient Intelligence, IOS Press. 
Barrué, C., Cortés, U., Martínez, A.B., Escoda, J., Annicchiarico, R. and Caltagirone, C. (2006) 'e-Tools: an agent coordination layer to support the mobility of persons with disabilities', in IFIP AI, pp.425-434.

Barrué, L., Céspedes, C., Cortés, U., Annicchiarico, R. and Caltagirone, C. (2004) 'e-Tools v1: the agent level', in Proc. of 2nd ECAI Workshop on Agents Applied in Health Care, pp.55-61.

Bourhis, G., Horn, O., Habert, O. and Pruski, A. (2001) 'An autonomous vehicle for people with motor disabilities', IEEE Robotics \& Automation Magazine, Vol. 8, pp.20-28.

Branch, L.G. and Jette, A.M. (1992) 'A prospective study of long-term care institutionalization among the aged', Am. J. Public Health, Vol. 72, pp.1373-1379.

Branting, L.K. and Aha, D.W. (1995) 'Stratified case-based reasoning: reusing hierarchical problem solving episodes', in Proceedings of the 14th International Joint Conference on Artificial Intelligence, pp.384-390.

Brooks, R.A. (1986) 'A robust layered control system for a mobile robot', IEEE Journal of Robotic and Automation, March, Vol. 2, pp.14-23.

Camarinha-Matos, L.M. and Afasarmanesh, H. (2001) Virtual Communities and Elderly Support, pp.279-284, WSES.

Carlesimo, G.A., Caltagirone, C. and Gainotti, G. (1996) 'The mental deterioration battery: normative data, diagnostic reliability and qualitative analyses of cognitive impairment. The group for the standardization of the mental deterioration battery', Eur. Neurol., Vol. 36, No. 6, pp.378-384.

Carmien, S., Dawe, M., Fischer, G., Gorman, A., Kintsch, A. and Sullivan, J.F. (2005) 'Socio-technical environments supporting people with cognitive disabilities using public transportation', Transactions on Human-Computer Interaction, Vol. 12, No. 2, pp.233-262.

Cheng, K.C., Bateni, H. and Maki, B.E. (2008) 'Reducing adverse effects of walkers on stability', in A. Mihailidis, J. Boger, H. Kautz and L. Normi (Eds.): Proc. Technology and Aging, 2007 International Conference on Technology and Aging, ICTA 2008, ATRS 21, pp.197-204, IOS Press, Amsterdam.

Chugo, D., Matsuoka, W., Jia, S., Takase, K. and Asama, H. (2008) 'Standing assistance system for rehabilitation walker', in B. Siciliano, O. Khatib and F. Groen (Eds.): Field and Service Robotics, Springer Tracts in Advanced Robotics, Vol. 42, pp.541-550, Springer-Verlag.

Confident Information Environment for the Independent Living of People with Severe Disabilities (Confident), available at http://212.73.32.174/Fundacion/Europeos/Confident/en/presentation/CF_TOCP.

Connell, J.H. and Viola, P. (1990) 'Cooperative control of a semi-autonomous mobile robot', in Proceedings of the IEEE Conference on Robotics and Automation, Cincinnati.

Cortés, U., Annicchiarico, R., Vázquez-Salceda, J., Urdiales, C., Cañamero, L., López, M., Sànchez-Marrè, M. and Caltagirone, C. (2003) 'Assistive technologies for the disabled and for the new generation of senior citizens: the e-Tools architecture', AI Communications, Vol. 16, pp.193-207.

Cortés, U., Martínez, A.B., Barrué, C., Benedico, T., Campana, F. and Annicchiarico, R. (2008) 'An agent-based service to elders mobility using the i-Walker', in the 7th International Conference on Autonomous Agents and Multiagent Systems (AAMAS08).

Czaja, S.J. and Lee, C.C. (2004) Designing Computer Systems for Older Adults, pp.413-427, Lawrence Erlbaum Associates.

Dochome: Remote Health Management Service for Patients with Chronic Heart Failure, available at http://www.fastuk.org/research/projview.php?id=936.

Espeland, M.A., Gill, T.M., Guralnik, J. et al. (2007) 'Designing clinical trials of interventions for mobility disability: results from the lifestyle interventions and independence for elders pilot (life-p) trial', J. Gerontol. A. Biol. Sci. Med. Sci., Vol. 62, No. 11, pp.1237-1243. 
Fernandez-Espejo, B., Poncela, A., Urdiales, C. and Sandoval, F. (2007) 'Collaborative emergent navigation based on biometric weighted shared control', in Proc. of the IWANN 2007, LNCS, Vol. 4507, pp.814-821.

Fried, L.P., Ferrucci, L., Darer, J., Williamson, J.D. and Anderson, G. (2004) 'Untangling the concepts of disability, frailty and comorbidity: implications for improved targeting and care', J. Gerontol. A. Biol. Sci. Med. Sci., Vol. 59, pp.255-263.

Fried, L.P., Tangen, C.M., Waltson, J., Newman, A.B. et al. (2001) 'Frailty in older adults: evidence for a phenotype', J. Gerontol. A. Biol. Sci. Med. Sci., Vol. 56A, pp.146-157.

Glover, J., Holstius, D., Manojlovich, M., Montgomery, K., Powers, A., Wu, J., Kiesler, S., Matthews, J. and Thrun, S. (2003) 'A robotically-augmented walker for older adults', Technical Report CMU-CS-03-170, Computer Science Department, Carnegie Mellon University, Pittsburgh, PA.

Gomi, T. and Griffith, A. (1998) 'Developing intelligent wheelchairs for the handicapped', Lecture Notes in Computer Science, Vol. 1458, pp.150-178, Springer-Verlag.

Graf, B., Hans, M. and Schraft, R.D. (2004) 'Care-o-bot II - development of a next generation robotic home assistant', Auton. Robots, Vol. 16, No. 2, pp.193-205.

Guralnik, J.M., LaCroix, A.Z., Abbot, R.D., Berkman, L.F., Satterfield, S., Evans, D.A. and Wallace, R.B. (1993) 'Maintaining mobility in late life, demographic characteristics and chronic conditions', Am. J. Epidemiology, Vol. 137, No. 8, pp.845-857.

Iezzoni, L., McCarthy, E., Davis, R. and Siebens, H. (2001) 'Mobility difficulties are not only a problem of old age', J. Gen. Intern. Med., Vol. 16, No. 4, pp.235-243.

Innovative GPRS/UMTS Mobile Services for Applications in Healthcare (mobihealth), available at http://www.mobihealth.org.

Inouye, S.K., Studenski, S., Tinetti, M.E. and Kuchel, G.A. (2007) 'Geriatric syndromes: clinical, research and policy implications of a core geriatric concept', J. Am. Geriatr. Soc., Vol. 55, pp.780-791.

ISO 9241-11 (1998) 'Ergonomic requirements for office work with visual display terminals (VDTS) part 11: guidance on usability', available at http://www.iso.org/iso/iso_catalogue/catalogue_tc/catalogue_detail.htm?csnumber=16883.

ITU/SPU Ubiquitous Network Societies, available at http://www.itu.int/osg/spu/ni/ubiquitous/.

Kamel-Boulos, M.N., Castellot Lou, R., Anastasiou, A., Nugent, C.D., Alexandersson, J., Zimmermann, G., Cortés, U. and Casas, R. (2009) 'Connectivity for healthcare and well-being management: examples from six European projects', Int. J. Environ. Res. Public Health, Vol. 6, No. 7, pp.1947-1971.

Katevas, N.L., Sgouros, N.M., Tzafestas, S.G., Papakonstantinou, G., Beattie, P., Bishop, J.M., Tsanakas, P. and Koutsouris, D. (1997) 'The autonomous mobile robot scenario: a sensor-aided intelligent navigation system for powered wheelchairs', IEEE Robotics Autom. Mag., Vol. 4, No. 4, pp.60-70.

Khatib, O. (1986) 'Real-time obstacle avoidance for manipulators and mobile robots', International Journal of Robotics Research, Vol. 5, No. 1, pp.90-98.

Kulyukin, V., Kutiyanawala, A., LoPresti, E., Matthew, J. and Simpson, R. (2008) 'i-Walker: toward a Rollator-mounted way-finding system for the elderly', in IEEE International Conference on RFID, pp.303-311, IEEE.

Lankenau, A. and Röfer, T. (2000) 'The role of shared control in service robots - the Bremen autonomous wheelchair as an example', in Service Robotics - Applications and Safety Issues in an Emerging Market, Workshop Notes, pp.27-31.

Likhachev, M., Kaess, M. and Arkin, R. (2002) 'Learning behavioural parameterization using spatiotemporal case-based reasoning', in Proc. of the 2002 IEEE International Conference on Robotics and Automation (ICRA), Vol. 2, pp.1282-1289. 
Mahoney, F.I. and Barthel, D.W. (1965) 'Functional evaluation: the Barthel index', Md. State. Med. J., Vol. 14, pp.61-65.

Mandel, C., Huebner, K. and Vierhuff, T. (2005) 'Towards an autonomous wheelchair: cognitive aspects in service robotics', in Proceedings of Towards Autonomous Robotic Systems (TAROS 2005), pp.165-172.

Marin, I., Arceredillo, E., Zuloaga, A. and Arias, J. (2005) 'Wireless sensor networks: a survey on ultra-low power-aware', Transactions on Engineering, Computing and Technology, Vol. 8, pp.44-49.

Martínez, A.B., Escoda, J., Benedico, T., Cortés, U., Annicchiarico, R., Barrué, C. and Caltagirone, C. (2005) 'Patient-driven mobile platform to enhance conventional wheelchair, with multi-agent system supervisory control', in Multi-Agent Systems and Applications IV: 4th International Central and Eastern European Conference on Multi-Agent Systems, CEEMAS 2005, Lecture Notes in Computer Science, Vol. 3690, pp.92-101, Springer-Verlag.

McCreadie, C. and Tinker, A. (2005) 'The acceptability of assistive technology to older people', Ageing and Society, Vol. 25, pp.91-110.

Miller, D.P. and Slack, M.G. (1995) 'Design \& testing of a low-cost robotic wheelchair', Autonomous Robots, Vol. 1, No. 3.

Nejatbakhsh, N. and Kosuge, K. (2005) 'User-environment based navigation algorithm for an omnidirectional passive walking aid system', pp.178-181.

Nikolaus, T., Specht-Leible, N., Bach, M., Oster, P. and Schlierf, G. (1999) 'A randomized trial of comprehensive geriatric assessment and home intervention in the care of hospitalized patients', Age and Ageing, Vol. 28, pp.543-550.

Patterson, D.J., Liao, L., Gajos, K., Collier, M., Livic, N., Olson, K., Wang, S., Fox, D. and Kautz, H. (2004) 'Opportunity knocks: a system to provide cognitive assistance with transportation services', in Proceedings of Ubi-Comp 2004.

Pollack, M.E. (2005) 'Intelligent technology for an aging population: the use of AI to assist elders with cognitive impairment', AI Magazine, Vol. 26, No. 2, pp.9-24.

Ram, A., Arkin, R.C., Moorman, K. and Clark, R.J. (1993) 'Case-based reactive navigation: a case-based method for on-line selection and adaptation of reactive control parameters in autonomous robotic systems', Technical Report GIT-CC-92/57, College of Computing, Georgia Institute of Technology.

Ramos, C., Augusto, J.C. and Shapiro, D. (2008) 'Ambient intelligence - the next step for artificial intelligence', IEEE Intelligent Systems, Vol. 23, No. 2, pp.15-18.

Rao, R.S., Conn, K., Jung, S.H., Katupitiya, J., Kientz, T., Kumar, V., Ostrowski, J., Patel, S. and Taylor, C.J. (2002) 'Human robot interaction: applications to smart wheelchairs', in Proceedings of the IEEE Conference on Robotics and Automation, Washington.

Rentscheler, A.J., Cooper, R.A., Blash, B. and Boninger, M.L. (2003) 'Intelligent walkers for the elderly: performance and safety testing of VA-PAMAID robotic walker', Journal of Rehabilitation Research and Development, Vol. 4, No. 5, pp.423-432.

Rodriguez-Losada, D., Matia, F., Jimenez, A., Galan, R. and Lacey, G. (2005) 'Implementing map based navigation in Guido, the robotic SmartWalker', in Proceedings of the 2005 IEEE International Conference on Robotics and Automation, pp.3390-3395.

Rogers, D.J. and Tanimoto, T.T. (1960) 'A computer program for classifying plants', Science, Vol. 132, No. 3434, pp.1115-1118.

Sánchez-Tato, I., del Toro, J.C., Pérez, E.J., Urdiales, C., Cortés, U., Annicchiaricco, R., Sandoval, F. and Caltagirone, C. (2007) 'Collaborative control for personalized mobility assistance to persons with physical/cognitive disabilities', in Proc. of IROS-2007 - Workshop on Assistive Technologies (IROS07).

Shneiderman, B. and Plaisant, C. (2005) Designing the User Interface: Strategies for Effective Human-Computer Interaction, Addison-Wesley. 
Simpson, R.C. (2005) 'Smart wheelchairs: a literature review', Journal of Rehabilitation Research \& Development, Vol. 42, No. 4, pp.423-436.

Simpson, R.C. and Levine, S.P. (1998) 'NavChair: an assistive wheelchair navigation system with automatic adaptation', Lecture Notes in Computer Science, pp.235-255, Springer-Verlag.

Simpson, R.C. and Levine, S.P. (2002) 'Voice control of a powered wheelchair', IEEE Trans. Neural Syst. Rehabil. Eng., Vol. 10, No. 2, pp.122-125.

Supported Human Autonomy for Recovery and Enhancement of Cognitive and Motor Abilities using Information Technologies (SHARE-it) (2007) Available at http://www.ist-shareit.eu.

Topinkova, E. (2008) 'Aging, disability and frailty', Ann. Nutr. Metab., Vol. 52, No. 1, pp.6-11.

Ubiquitous Care System to Support Independent Living (Confidence), available at http://www.confidence-eu.org/.

Ultra wideband (UWB) Technology, available at http://www.intel.com/technology/comms/uwb/.

Urdiales, C., Pérez, E.J., Vázquez-Salceda, J., Sànchez-Marrè, M. and Sandoval, F. (2006) 'A purely reactive navigation scheme for dynamic environments using case-based reasoning', Autonomous Robots, Vol. 39, No. 5, pp.67-78.

Urdiales, C., Peula, J.M., Cortés, U., Barrué, C., Fernández-Espejo, B., Annichiaricco, R., Sandoval Hernández, F. and Caltagirone, C. (2009) 'A metrics review for performance evaluation on assisted wheelchair navigation', in IWANN, Vol. 1, pp.1145-1152.

Vanhooydonck, D., Demeester, E., Nuttin, M. and Van Brussel, H. (2003) 'Shared control for intelligent wheelchairs: an implicit estimation of the user intention', in Proceedings of the 1st International Workshop on Advances in Service Robotics 2003.

Vescovo, F. (2005) 'Casa Agevole', available at http://www.progettarepertutti.org/progettazione/casaagevole-fondazione/index.html.

Wasson, G., Gunderson, J., Graves, S. and Felder, R. (2001) 'An assistive robotic agent for pedestrian mobility', in Int. Conf. on Autonomous Agents, pp.169-173, ACM.

Wasson, G., Sheth, P., Alwan, M., Huang, C. and Ledoux, A. (2003) 'User intent in a shared control framework for pedestrian mobility aids', in IEEE/RSJ International Conference on Intelligent Robots and Systems (IROS), pp.2962-2967, IEEE.

Wasson, G., Sheth, P., Huang, C. and Alwan, M. (2007) 'Shared navigational control and user intent detection in an intelligent walker', Aging Medicine, pp.53-76, Springer-Verlag.

Weiser, M. (1991) 'The computer for the twenty-first century', Scientific American, September, pp.94-104.

Yanco, H.A. (1998) 'Wheelesley: a robotic wheelchair system: indoor navigation and user interface', Assistive Technology and Artificial Intelligence, Applications in Robotics, User Interfaces and Natural Language Processing, pp.256-268.

\section{Notes}

1 In this case, we do not use case adaptation so as not to modify original values from patients. 